Paediatr. Paedolog. 2020 · 55:104-105 https://doi.org/10.1007/s00608-020-00805-1 Online publiziert: 4. Juni 2020

(c) Springer-Verlag GmbH Austria, ein Teil von Springer Nature 2020

Die Kinder- und Jugendheilkunde gehört zu den medizinischen Disziplinen mit einem außerordentlich hohen Anteil an unterschiedlichen und teilweise seltenen Krankheitsbildern, die ein umfassendes Verständnis für die oft in verschiedenen Organsystemen und Altersgruppen auftretenden Erkrankungen erfordern. Diese Vielfalt unseres Fachgebiets ist auch dem Inhalt dieser Ausgabe von Pädiatrie \& Pädologie zu entnehmen. Mit eben dieser zunehmenden Komplexität unseres Fachgebiets wird es für viele junge Pädiater attraktiver, sich in der Praxis nicht nur auf einen fachlichen Schwerpunkt zu stützen, sondern vielmehr Kooperationsformen mit anderen Kolleginnen und Kollegen anzudenken.

Der zugrundeliegende Gedanke, mehrere Ärzte sowie ausreichend spezialisiertes medizinisches Fachpersonal und eine fachlich hochwertige technische Ausrüstung in einer Einheit anzubieten, bringt erhebliche Vorteile: Die Ärzte in der Kooperation ergänzen sich inhaltlich, dadurch wird der Austausch gefördert und das medizinische Angebot verbreitert, und damit kann die Qualität der Versorgung auf einem hohen Niveau gehalten werden. Gerade junge Fachärzte, die von dieser fachlichen Breite oft noch verunsichert sind, schätzen die Sicherheit der Arbeit im Team. Darüber hinaus können die unterschiedlichen Persönlichkeiten der tätigen Ärzte das Angebot bereichern. Allerdings stellt sich die Frage, in welcher Organisationsform eine derartige Kooperation am besten gelebt werden kann.

\title{
Peter Voit
}

First Vienna Pediatric Medical Center, Erstes Wiener Kindergesundheitszentrum Donaustadt, Sigmund Freud Universität Wien, Wien, Österreich

\section{Pädiatrische Versorgungszentren: Vorbild Deutschland?}

In Deutschland sind seit dem Jahr 2003 sogenannte Medizinische Versorgungszentren (MVZ) etabliert. Ein MVZ ist in Deutschland eine fachübergreifende, ärztlich geleitete Einrichtung, in der im Arztregister eingetragene Ärzte als Inhaber (Vertragsärzte) oder als Angestellte tätig sind. Medizinische Versorgungszentren nehmen an der vertragsärztlichen Versorgung teil.

Gesellschafter eines MVZ können zugelassene Leistungserbringer nach dem Sozialgesetzbuch sein, also auch Krankenhäuser, Ärzte, Zahnärzte, Psychotherapeuten, Apotheker, Pflegedienste, Rehabilitationseinrichtungen, Zahntechniker oder Hebammen. Dies ist ein bedeutender Unterschied zu den Primärversorgungseinrichtungen (PVE) in Österreich, an denen keine Kapitalgesellschaften beteiligt sein können.

Die Einführung von Versorgungszentren würde einen erheblichen Strukturwandel in der vertragsärztlichen Versorgung bewirken. Chancen entstehen hier beispielsweise in der Verkürzung von Durchlaufzeiten, Verminderung von Schnittstellen für den Patienten sowie Verbesserung von Effizienz und Kundenorientierung. Die Ärztekammer hat diese wiederholt mit den Polikliniken der ehemaligen DDR verglichen und als Negativbeispiel präsentiert.

Für die Patienten ist ein MVZ mit einer Gemeinschaftspraxis vergleichbar. Im Gegensatz zur bisher durch Vertragsärzte oder Wahlärzte sichergestellten ambulanten Versorgung entsteht kein Behandlungsvertrag mit dem Arzt, sondern mit dem MVZan sich. Der Patient hat keinen Anspruch auf persönliche Behandlung durch einen der Ärzte im MVZ. Medizinische Versorgungszentren wer- den auch oft als Schritt gesehen, die bisherige Trennung der ambulanten und stationären Versorgung aufzuheben.

Fachübergreifend ist ein MVZ dann positioniert, wenn mindestens zwei verschiedene Facharztgruppen vertreten sind. Ökonomisch betrachtet bedeutet diese Regelung, dass der Vertragsarzt nicht nur durch eigene ärztliche Leistungen Geld verdienen kann, sondern auch dadurch, dass er unternehmerisch, also durch Anstellung anderer Ärzte, wirtschaftlichen Erfolg erzielen kann. Das würde eine Neuerung im medizinischen Honorierungssystem darstellen, da die Honorierung von niedergelassenen Ärzten bislang in der Regel aus eigener Arbeit bestanden hat.

MVZ gibt es sowohl in städtischen als auch ländlichen Regionen, allerdings besteht die Mehrzahl der MVZ in Gebieten mit einer hohen Einwohnerzahl, auf dem Land ist eine Unterversorgung bemerkbar. An der überwiegenden Zahl der MVZ sind Vertragsärzte und Krankenhäuser als Träger beteiligt, Hausärzte und Internisten sind die am häufigsten vertretenen Fachgruppen in den MVZ.

Es bestanden Ende 2015 insgesamt 2156 MVZin Deutschland, die 12.976 angestellte und 1341 freiberufliche Ärzte beschäftigten [1]. Medizinische Versorgungszentren basieren in der Regel auf der Gesellschaftsform der GmbH.

Als neue Leistungserbringer müssen die MVZ alle erforderlichen personellen und sachlichen Mittel selbst bereitstellen. Das stellt hohe Anforderungen an das Zentrumsmanagement, das zum einen den effizienten, reibungslosen und wirtschaftlichen Betrieb sicherzustellen hat, zum anderen aber auch den Personaleinsatz, die Einhaltung der Behand- 
lungsprozesse sowie die Auslastung des Zentrums steuern muss. Die damit verbundene Sorge um eine zu starke Ökonomisierung der Medizin ist nicht von der Hand zu weisen.

Hauptkritikpunkte an der Versorgung durch MVZ sind daher auch, dass das Eindringen von Managementgesellschaften, privaten Klinikträgern und Krankenhäusern in den ambulanten Versorgungsmarkt die freie Arztwahl der Patienten einschränke, die Freiberuflichkeit der ärztlichen Tätigkeit gefährde und niedergelassene Facharztpraxen verdränge.

Medizinische Versorgungszentren oder PVE können also in Summe dann eine durchaus interessante Option in der zukünftigen multidisziplinären Versorgung darstellen, wenn eine Integration in die bestehende Versorgungslandschaft gelingt und eine Kommerzialisierung durch Investoren vermieden werden kann.

Die angesprochene multidisziplinäre Breite unseres Fachgebiets findet sich auch in dieser Ausgabe wieder. Dazu gehören Hörstörungen, für deren erfolgreiche Behandlung es wichtig ist, diese zeitgerecht $\mathrm{zu}$ vermuten und ei- ner gezielten Diagnostik zuzuführen. Wenn indiziert, kann frühzeitig eine entsprechende Behandlung mittels Cochlearimplantat angeboten werden; diesem Thema ist ein Beitrag gewidmet. Das Projekt „KidsAP“ behandelt ein neues System einer künstlichen Bauchspeicheldrüse für die Behandlung von Diabetes Typ I bei Kleinkindern bis zum Volksschulalter. Dieses Closed-LoopSystem kann via Handy-App gesteuert werden und bietet die Möglichkeit einer Verbesserung der Lebensqualität dieser Patienten. Ein häufiges Phänomen in der Praxis stellt die Frage nach der Indikation einer Supplementierung von Vitamin D dar, wofür praxisgerechte Empfehlungen benötigt werden.

In diesem Sinn wünsche ich viel Freude bei der Lektüre dieser Ausgabe!

\section{Peter Voitl}

\section{Korrespondenzadresse

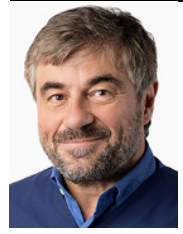 \\ (C) P. Voitl \\ Prim. MedR. Ass.-Prof. DDr. Peter Voitl, MBA \\ First Vienna Pediatric Medical Center, Erstes Wiener \\ Kindergesundheitszentrum \\ Donaustadt, Sigmund Freud Universität Wien Donaucitystraße 1, 1220 Wien, Österreich office@kinderarzt.at}

Interessenkonflikt. P. Voitl gibt an, dass kein Interessenkonflikt besteht.

\section{Literatur}

1. Kassenärztliche Bundesvereinigung (2015) Entwicklungen der Medizinischen Versorgungszentren. Kassenärztliche Bundesvereinigung, Berlin

Hinweis des Verlags. Der Verlag bleibt in Hinblick auf geografische Zuordnungen und Gebietsbezeichnungen in veröffentlichten Karten und Institutsadressen neutral.

Hier steht eine Anzeige. 